\title{
Parameter Estimation for Ridge Detection in Images with Thin Structures
}

\author{
Talita Perciano ${ }^{1, \star}$, Roberto Hirata $\mathrm{Jr}{ }^{1}$, and Lúcio André de Castro Jorge ${ }^{2}$ \\ 1 Instituto de Matemática e Estatística, Universidade de São Paulo, \\ São Paulo, SP 05508-090, Brazil \\ \{talitap, hirata\}@ime.usp.br \\ 2 CNPDIA, Embrapa Instrumentação Agropecuária, São Carlos, \\ SP 13560-970, Brazil \\ lucio@cnpdia.embrapa.br
}

\begin{abstract}
This paper presents an analysis of four ridge detectors in images with thin structures: plant root images and retinal images. Two proposed detectors and two detectors from the literature are used. We estimate the optimal parameters for each detector for the two applications using a ROC curve similar approach. Simulated images of plant roots and retinal images are used. The optimal parameters are estimated and then used in real images. We conclude that the proposed detector based on mathematical morphology and the one based on the steerable filter are the best for both set of images.
\end{abstract}

Keywords: Ridge detection, parameter estimation.

\section{Introduction}

The segmentation of complex images is one of the hardest tasks in image processing. We consider complex images that have one or more of the following characteristics: (1) thin and elongated structures (relative to the image dimensions) eventually with ramified body, (2) subpixel structures, (3) missing borders (lack of information to fully segment the image). The objective of the study presented here is to analyze some ridge detectors and to find optimal parameters for them using a ROC curve similar approach. We used simulated images of soil profiles with plant roots generated by an existent software [15] and retinal images from the public database DRIVE (Digital retinal images for vessel extraction [17]).

We want to develop algorithms sensible to this kind of structures. Efficient solutions may be used in many applications: segmentation of river and road networks in SAR images [18, segmentation of trees, blood vessels [16], neurons [4] and plant roots (important application in agriculture research). We are not aware of any work in the literature which tackles the problem of segmenting plant roots, except of a procedure described in [14] (in Portuguese).

^ Thanks to FAPESP, CNPq and CAPES for funding.

I. Bloch and R.M. Cesar, Jr. (Eds.): CIARP 2010, LNCS 6419, pp. 386-393, 2010.

(C) Springer-Verlag Berlin Heidelberg 2010 
The problem of detecting ridges is well studied but we did not find any study on using information of thin structures to solve it. In [8], two methods for identification and analysis of the multiresolution behavior of ridges are described. In [10, two techniques of orientation analysis are explored for the detection of oriented structures like ridges. A new framework for automatic local control of scale levels for ridge detection is presented in 12. In [13, the authors review some characterizations to formalize the notion of ridges and propose a new method. The detection of ridges was also used in [17] for the development of an automatic segmentation technique of vessels in retinal images. A very recent work in the literature explores methods for ridge detection [1].

This paper makes the analysis of ridge detection in images with thin structures, specifically retinal and plant root images. Four techniques are explored: a modified Canny detector, a morphological approach, the Frangi filter (see [7,6]) and a steerable filter [11. ROC curves are used to analyze these techniques and to find their optimal parameters. In Section 2, we describe two simple proposed ridge detectors and we briefly describe the two other detectors used from the literature. In Section 3, we explain the methodology used to estimate optimal parameters for the detectors. In Section 4 , the experimental results are presented. The conclusions in Section 5 synthesize the results of the paper.

\section{Ridge Detection}

\subsection{Proposed Detectors}

The following techniques for identifying thin structures are based on the fact that: (i) the detectors are applied directly to the image, avoiding the loss of detailed information (i.e., only filters that preserve borders and ridges can be applied before the method that extracts ridges/edges); (ii) The aim is to detect the highest quantity of ridges as possible that correspond to plant roots (or vessels). The problem of false detection is not tackled in this stage of the project.

Canny Ridge Detector. The Canny edge detector [3] is one of the most known and used in image processing and computer vision because its performance is superior to other detectors in many situations, despite of being more complex [9]. This detector has four steps: (i) smooth the input image to reduce the noise; (ii) compute the gradient and the gradient angles of the image; (iii) apply nonmaxima suppression to the gradient of the image; (iv) use double thresholding and connective analysis to detect and to link edges.

Canny defined an edge detector but not a ridge detector. Taking into account the two considerations made in the beginning of this section, it was necessary to modify the Canny algorithm in order to make the method detect the ridges. The first change is to remove step i), i.e., the original image is not smoothed. Another change is to use the original image instead of the gradient of the image (because ridges are the main objective) in steps ii) and iii).

Considering the image as a surface, the points of local maxima of curvature are localized in the center of the thin structures and the result of step 4 is equivalent 
(not equal) to a grayscale skeleton [5]. Using this variation of the algorithm, the information of the location of the ridges is not lost (Section 4).

Detection Using Mathematical Morphology. Another technique used to detect ridges from the original image is based on a composition of morphological operators. This is also a variation of top-hat algorithms found in the literature.

The mathematical morphology concepts used by the method are not presented here because the lack of space, however they can be found in [5].

Three steps are used to detect the ridges of an input image:

1. Application of the opening by reconstruction top-hat; (mmopenrecth)

2. Thresholding of the image resulting from the first step; (mmthreshad)

3. Homotopic skeleton by thinning to obtain the final result. (mmthin)

One important variable parameter for this detector is the threshold. It controls the level of precision when detecting the peaks in the image. The higher the threshold, the lower the number of peaks detected.

The Python script below makes the ridge detection of the an image.

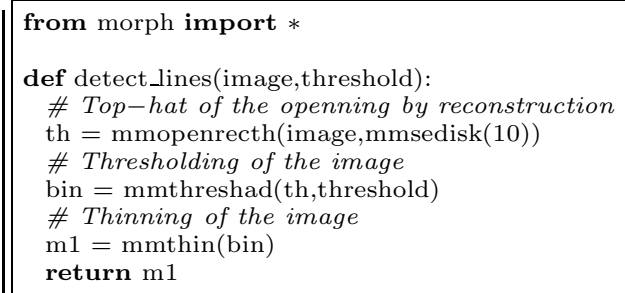

\subsection{The Frangi Filter}

The Frangi filter is actually a Hessian-based vessel enhancement method proposed by [7. In the literature it is assumed that the intensity profile of a vessel in the cross section can be modeled by a Gaussian function and that the intensity does not change much along vessels. In order to distinguish vessels from other structures, second order derivative features such as curvatures in Hessian-based enhancement filters are used. Vessels have small curvature along their center lines and large curvature in the sectional direction. The two principal curvatures can be acquired from the Hessian matrix (eigenvalues), $\lambda_{1}$ and $\lambda_{2}$. Frangi computes the scores $\mathcal{R}_{\mathcal{B}}=\left|\lambda_{1}\right| /\left|\lambda_{2}\right|$ and $\mathcal{S}=\sqrt{\lambda_{1}^{2}+\lambda_{2}^{2}}$ and define the response of his filter for $2 \mathrm{D}$ vessel as:

$$
\mathcal{V}_{o}(s)= \begin{cases}0 & \text { if } \lambda_{2}>0, \\ \exp \left(-\frac{\mathcal{R}_{\mathcal{B}}^{2}}{2 \beta^{2}}\right)\left(1-\exp \left(-\frac{\mathcal{S}^{2}}{2 c^{2}}\right)\right), & \text { otherwise }\end{cases}
$$

where parameters $\beta$ and $c$ are constants. The result of this filter is a probability map of the pixels being vessels. In order to find the medial axis of the vessels we apply a threshold followed by a thinning operator to this probability map. 


\subsection{Steerable Filter}

The steerable filters [1] are 2D feature detectors, i.e., a class of steerable functions based on the optimization of a Canny-like model. These filters have closedform expressions and lead to operators that have a better orientation selectivity than classical Hessian-based detectors. In order to obtain a ridge detector, high order derivates of the Gaussian are used. In the present case the 4th order is adopted. A threshold has to be applied to the final result of the image to obtain a binary image with the ridges and non-ridges map. The detailed explanation of these filters are out of the scope of this work. An implementation of these filters is available as a plugin for the famous ImageJ software.

\section{Parameter Estimation}

We can use various methods of ridge detection and typically these methods have some parameters that can be changed, like the ones presented in the previous section. A precision analysis using a similar idea to the ROC curves allows us to adjust the parameters associated with the methods, choosing optimal values for them. In order to make the analysis, it is necessary to have the ground-truths of the images the detector was applied to. Therefore, we used retinal images from the DRIVE database (with ground-truths made by specialists) and simulated plant root images. The ground-truths of the images are for segmentation purposes, thus we apply a thinning operator to them in order to obtain the optimal ridge maps.

For each set of images we executed a simplified version of the process described by [2. Therefore, we were able to find the best parameters for each ridge detector. The process consists of the following steps:

1. First, the minimum and maximum values for each parameter of the detectors are chosen. The range of the parameters were found empirically, as we can find the values of the extreme cases (totally white/black images).

2. At the beginning, the intervals of each parameter are uniformly divided to generate four values for each one.

3. From the intervals with four parameters, it is possible to make some refinements:

- For two successive values in the interval, it is generated a new value that is the half of the subinterval between these two values. So, let an initial interval for a detector be $4 \times 4 \times 4$, i.e., four values for each parameter. After the application of the refinement for the first parameter, for instance, we would have the interval $7 \times 4 \times 4$.

The intervals are refined until the improvement of the curve is minimum.

4. Now we obtain a curve that is similar to a ROC curve. The detectors are applied for each possible parameter set obtained from the refinements resulted from the above step.

5. Finally, the median of the curves of all the images is calculated (ten images in this case). 
In the end of the process, a curve for each detector is obtained. From these curves it is possible to find the optimal parameters for each one. The chosen parameters are those that lead to the highest True Positive Rate (TPR) value with acceptable values of false positives. It is important to notice that the proposed ridge detectors do not achieve totally white images (the case that all pixels would be classified as roots/vessels). However, as the Frangi filter and the steerable filter results are based on thresholding a probability map, it is clear that, in some cases the results will tend to totally white images. In such cases, we choose as the best parameter those that lead to the highest TPR with the value of False Positive Rate (FPR) related to the TPR values obtained for the other two detectors.

\section{Experimental Results}

The process above was applied to ten simulated root images and to ten retinal images. The median ROC curves for each set of images and for each detector are presented in Figure 1 .

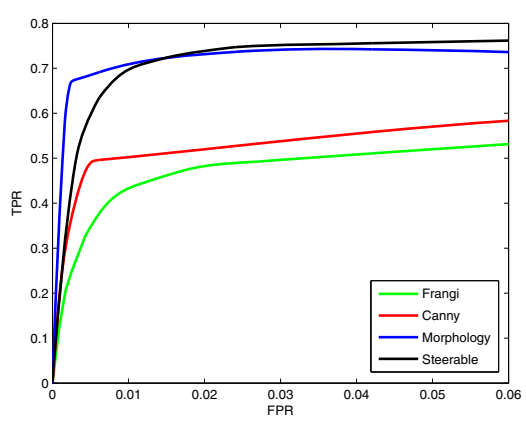

(a) Simulated root images

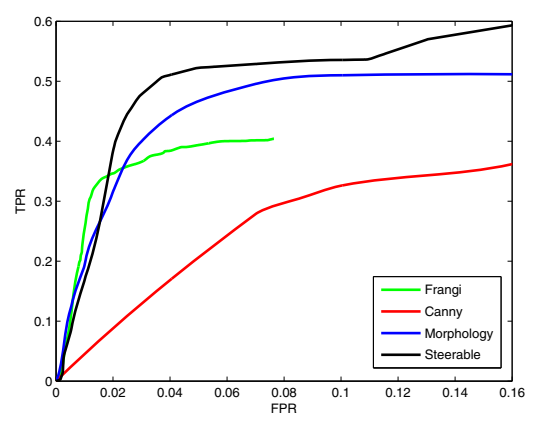

(b) Retinal images

Fig. 1. Median ROC curves for each application and each detector

After the previous analysis, the optimal parameters are obtained for the simulated root images and for the retinal images which are presented in Table 1.

Figures 2 and 3 present the results of the detectors for a simulated root image and a retinal image, respectively, using the optimal parameters.

The optimal parameters obtained for the simulated root images were also used experimentally for the real root images. We did not have the ground-truths of the real images of plant roots and that is why we used the parameters of the synthetic images. Figure 4 presents the results for one of the images.

Table 2 shows the summary results for each ridge detector analyzed in this paper. The set of images from where the TPR and the FPR were obtained is 
Table 1. Optimal parameters for both applications and each filter

\begin{tabular}{|c|c|c|c|c|c|c|}
\hline \multirow{2}{*}{$\frac{\text { Detectors }}{\text { Canny }}$} & \multicolumn{3}{|c|}{ Plant root Images } & \multicolumn{3}{|c|}{ Retinal Images } \\
\hline & $\mid T_{L}=0.33$ & $T_{H}=1$ & $|\sigma=1.2292|$ & $\left|T_{L}=0.1666\right|$ & $T_{H}=1$ & $\sigma=9.375$ \\
\hline Morphology & \multicolumn{3}{|c|}{$T s=26.3541$} & \multicolumn{3}{|c|}{$T s=1.0436$} \\
\hline Frangi & \multicolumn{3}{|c|}{$\beta=11 \quad \mid T=1 \times 10^{-5}$} & \multicolumn{3}{|c|}{$T=1 \times 10^{-6}$} \\
\hline Steerable & \multicolumn{3}{|c|}{$T=51$} & \multicolumn{3}{|c|}{$T=14$} \\
\hline
\end{tabular}

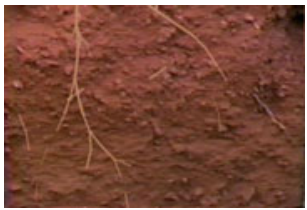

(a) Original image

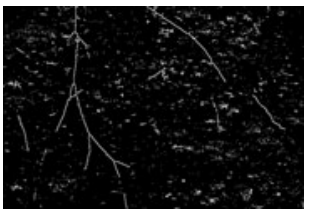

(d) Morphplogy

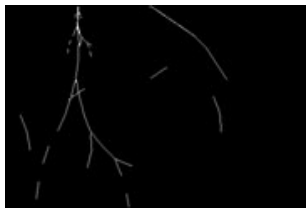

(b) Ground-truth

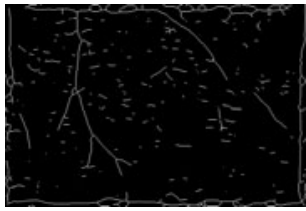

(e) Frangi

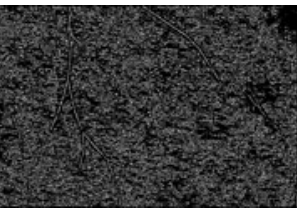

(c) Canny

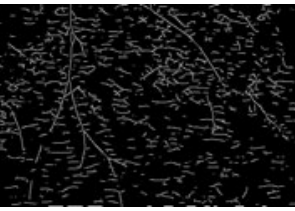

(f) Steerable

Fig. 2. Results applied to a simulated plant root image using optimal parameters

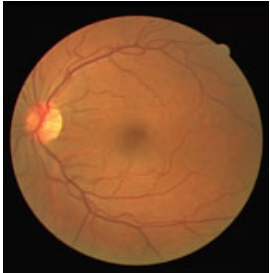

(a) Original image

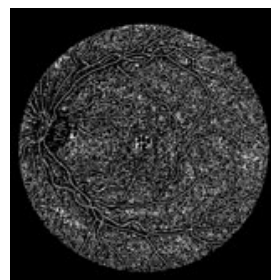

(d) Morphology

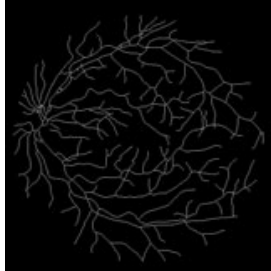

(b) Ground-truth

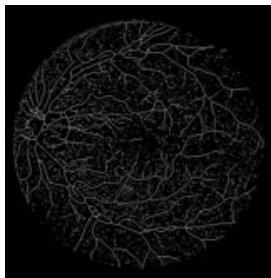

(e) Frangi

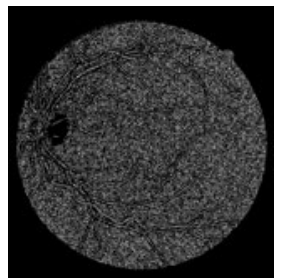

(c) Canny

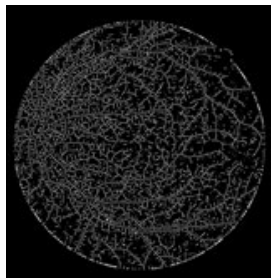

(f) Steerable

Fig. 3. Detectors results applied to a retinal image using optimal parameters 


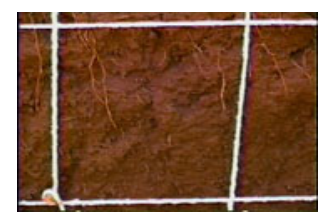

(a) Original image

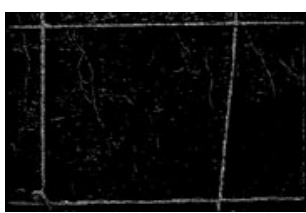

(b) Canny

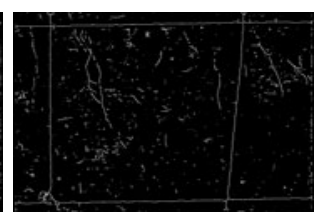

(c) Morphology

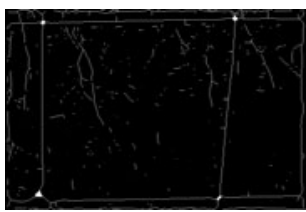

(d) Frangi

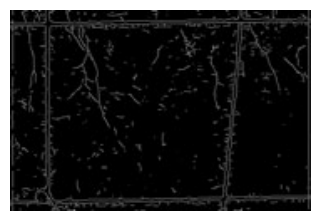

(e) Steerable

Fig. 4. Detectors results applied to a real root image

Table 2. Values for TPR and FPR for all detectors

\begin{tabular}{l|lccc}
\hline \multirow{2}{*}{ Detectors } & \multicolumn{4}{|c}{ Plant root images Retinal images } \\
& TPR & FPR & TPR & FPR \\
\hline \hline Canny & 0.58 & 0.24 & 0.44 & 0.19 \\
\hline Morphology & 0.74 & 0.049 & 0.51 & 0.16 \\
\hline Frangi filter & 0.47 & 0.025 & 0.46 & 0.09 \\
\hline Steerable filter & 0.76 & 0.052 & 0.58 & 0.14 \\
\hline
\end{tabular}

different from the one used to obtain the optimal parameters. We can conclude that the best detectors for both set of images are the one based on mathematical morphology and the one based on the steerable filter. Despite of the simplicity of the morphology detector proposed, it obtained very comparable results with the steerable detector. These results can be also visually noticed in the images shown in Figure 2 .

\section{Conclusions}

This work presented a study of ridge detectors in plant root images and retinal images using ROC curves aiming to obtain optimal parameters for each detector and for each application. The optimal parameters are estimated and applied to real images. The comparison among the detectors, using visual and quantitative results, shows that the best ridge detectors are the one based on mathematical morphology and the steerable filter. This study is part of a larger project where ridge detectors are being used as a basic representation for the development of segmenation techniques applied to images with thin structures. 


\section{References}

1. Berlemont, S., Olivo Marin, J.C.: Combining local filtering and multiscale analysis for edge, ridge, and curvilinear objects detection. IEEE Transactions on Image Processing 19(1), 74-84 (2010)

2. Bowyer, K., Kranenburg, C., Dougherty, S.: Edge detector evaluation using empirical roc curves. Comput. Vis. Image Underst. 84(1), 77-103 (2001)

3. Canny, J.: A computational approach to edge detection. IEEE Trans. Pattern Anal. Mach. Intell. 8(6), 679-698 (1986)

4. Dima, A., Scholz, M., Obermayer, K.: Automatic segmentation and skeletonization of neurons from confocal microscopy images based on the 3-d wavelet transform. IEEE Transactions on Image Processing 11(4), 790-801 (2002)

5. Dougherty, E.R., Lotufo, R.A.: Hands-on Morphological Image Processing. SPIE Publications, Bellingham (2003)

6. Frangi, A.F.: Three-dimensional Model-based Analysis of Vascular and Cardiac Images. Ph.D. thesis, Utrecht University, The Netherlands (2001)

7. Frangi, A.F., Niessen, W.J., Vincken, K.L., Viergever, M.A.: Multiscale vessel enhancement filtering, p. 130+ (1998), http://www.springerlink.com/content/84rpbx096y455vtv

8. Gauch, J.M., Pizer, S.M.: Multiresolution analysis of ridges and valleys in greyscale images. IEEE Trans. Pattern Anal. Mach. Intell. 15(6), 635-646 (1993)

9. Gonzalez, R.C., Woods, R.E.: Digital Image Processing, 3rd edn. Prentice-Hall, Englewood Cliffs (2008)

10. Hou, J., Bamberger, R.: Orientation selective operators for ridge, valley, edge, and line detection in imagery. In: IEEE International Conference on Acoustics, Speech, and Signal Processing, vol. 2, pp. 25-28 (1994)

11. Jacob, M., Unser, M.: Design of steerable filters for feature detection using cannylike criteria. IEEE Transactions on Pattern Analysis and Machine Intelligence 26, 1007-1019 (2004)

12. Lindeberg, T.: Edge detection and ridge detection with automatic scale selection. International Journal of Computer Vision 30, 465-470 (1996)

13. Lopez, A.M., Lumbreras, F., Serrat, J., Villanueva, J.J.: Evaluation of methods for ridge and valley detection. IEEE Transactions on Pattern Analysis and Machine Intelligence 21(4), 327-335 (1999)

14. Neto, L.M., Vaz, C.M.P., Crestana, S.: Instrumentação avançada em ciência do solo, 1st edn. EMBRAPA (2007)

15. Perciano, T., Hirata, R., Cesar, R.M.: An image simulator of soil profiles with plant roots for image segmentation. In: Pedrini, H., ao Marques de Carvalho, J., Lewiner, T. (eds.) Workshops of Sibgrapi 2009 - Posters, SBC, Rio de Janeiro, RJ (2009), http://www.matmidia.mat.puc-rio.br/Sibgrapi2009

16. Soares, J.V.B., Leandro, J.J.G., Cesar Jr., R.M., Jelinek, H.F., Cree, M.J.: Retinal vessel segmentation using the 2-D Gabor wavelet and supervised classification. IEEE Transactions on Medical Imaging 25, 1214-1222 (2006)

17. Staal, J., Abramoff, M., Niemeijer, M., Viergever, M., van Ginneken, B.: Ridgebased vessel segmentation in color images of the retina. IEEE Transactions on Medical Imaging 23(4), 501-509 (2004)

18. Tupin, F., Houshmand, B., Dactu, M.: Road detection in dense urban areas using SAR imagery anf the usefulness of multiple views. IEEE Trans. Geosci. Remote Sensing 40(11), 2405-2414 (2002) 